\title{
LA PERCEPCIÓN DEL RIESGO Y SU IMPLICACIÓN EN LA GESTIÓN Y PROMOCIÓN DE LOS DESTINOS TURÍSTICOS. EL EFECTO MODERADOR DEL DESTINO
}

\author{
Rita R. Carballo* \\ Universidad de Las Palmas de Gran Canaria \\ https://orcid.org/0000-0001-9847-7373 \\ María M. Carballo* \\ Universidad de Las Palmas de Gran Canaria \\ https://orcid.org/0000-0003-4489-3763 \\ Carmelo J.León* \\ Universidad de Las Palmas de Gran Canaria \\ https://orcid.org/0000-0001-9451-4093 \\ Sergio Moreno-Gil* \\ Universidad de Las Palmas de Gran Canaria \\ https://orcid.org/0000-0001-6905-8073
}

\section{RESUMEN}

El propósito de este estudio es examinar como contribuyen los distintos tipos de riesgos (terrorismo, accidente, delincuencia, salud, catástrofes) a los que se enfrenta los turistas a la percepción de riesgo final sobre los destinos. Asimismo, el trabajo analiza el efecto moderador del destino en las relaciones causales establecidas entre los tipos de riesgos y el riesgo percibido. Para ello se comparan dos grupos de destinos: Europa (Islas Canarias, España) y Latinoamérica, con los destinos de Brasil y Colombia. El trabajo se realizó mediante encuestas online a través de una empresa especializada en los mercados potenciales de Alemania y Reino Unido. El análisis se llevó a cabo mediante un modelo de ecuaciones estructurales (SEM) para probar las relaciones causales planteadas. Aunque la percepción de seguridad y protección y su influencia en el comportamiento del turismo es importante para la promoción y gestión de las actividades turísticas, su análisis empírico ha recibido

Fecha de recepción: 31 de mayo de 2019

Fecha de aceptación: 9 de enero de 2020

*Instituto Universitario de Turismo y Desarrollo Económico Sostenible-TIDES. Universidad de Las Palmas de Gran Canaria. Edificio D3. 09. Campus de Tafira. 35017 LAS PALMAS DE GRAN CANARIA (España). E-mail: rita.carballo@ulpgc.es, yalecafu@yahoo.es, carmelo.leon@ulpgc.es, sergio.moreno@ulpgc.es. 
una atención limitada entre los investigadores. Los resultados de este trabajo muestran interesantes conclusiones prácticas y académicas para las organizaciones empresariales y la promoción de los destinos turísticos.

Palabras clave: riesgo percibido; comunicación; organizaciones; gestión; ecuaciones estructurales.

\title{
The perception of risk and its implication in the promotion and tourist destinations management. The moderating effect of destination
}

\begin{abstract}
The purpose of this study is to examine how the different types of risks (terrorism, accident, crime, health, catastrophes) that tourists face contributes to the perception of final risk on destinations. Likewise, the paper analyzes the moderating effect of destination in the causal relationships established between the types of risks and the perceived risk. For this, two groups of destinations are compared: Europe (Canary Islands, Spain) and Latin America, with the destinations of Brazil and Colombia. The work was carried out through online surveys through a company specialized in the potential markets of Germany and the United Kingdom. The analysis was carried out using a model of structural equations (SEM) to prove the causal relationships raised. Although the perception of safety and protection and its influence on the behavior of tourism is important for the promotion and management of tourism activities, its empirical analysis has received limited attention among researchers. The results of this work show interesting practical and academic conclusions for business organizations and the promotion of tourist destinations.
\end{abstract}

Keywords: perceived risk; communication; organizations; management; structural equations model.

\section{INTRODUCCIÓN}

La paz y la seguridad son los principales condicionantes para el normal desarrollo turístico de un destino, región o país, y por lo tanto son los factores básicos determinantes de su crecimiento. Los turistas no sólo seleccionan un destino vacacional en base a la imagen y el precio, sino que existe un factor imprescindible que es la percepción de la seguridad.

La seguridad en un destino es uno de los aspectos más importantes a la hora de ofrecer a los turistas una experiencia de calidad (Otto y Ritchie, 1996). La UNWTO incluye la seguridad y protección como uno de los seis estándares determinantes de la calidad del producto turístico. La seguridad es un factor condicionante del atractivo de un destino e importante para promover el desarrollo turístico sostenible. Sin ella, los destinos no pueden competir con éxito, aunque presenten en sus campañas de marketing las cualidades más atractivas que posean, ya sean naturales o creadas. Por este motivo, los destinos 
prestan cada vez más una mayor atención a este tema. La seguridad en el turismo es un asunto global y concierne a todos los países, incluidos los desarrollados.

Son muchos y muy diversos los riesgos a los que se enfrenta un turista. Entender el proceso de formación del riesgo percibido es importante porque influye tanto en la imagen del destino como en el comportamiento del turista. Y por lo tanto puede ayudar en el diseño de prácticas para mejorar la imagen del destino e incrementar la demanda turística. Surge así la necesidad de profundizar en el conocimiento de los factores que conforman la percepción de riesgo que el turista tiene de un destino.

El presente artículo pretende contribuir a la literatura existente, plateándose dos objetivos. Inicialmente, se establecen a través de SEM las relaciones causales entre diversos tipos de riesgos (salud, accidente, terrorismo, catástrofes, delincuencia) y el riesgo percibido por el turista potencial en tres destinos turísticos internacionales (Canarias, Colombia y Brasil). Posteriormente se realiza un análisis multigrupo para examinar el efecto moderador que la variable "destino" pueda ejercer en las relaciones causales propuestas. Es decir, se analiza si los tipos de riesgos presentan distinta importancia dependiendo del destino que se visite. Para ello se divide la muestra en dos subgrupos de destinos: Europa (Islas Canarias, España) y Latinoamérica (Colombia y Brasil).

\section{REVISIÓN DE LA LITERATURA}

El turismo está generalmente asociado con el placer y actividades de ocio, y el riesgo es visto principalmente como algo que debe evitarse o esconderse como una amenaza potencial (Williams y Baláž, 2014). Pero turismo y riesgo están inherentemente relacionados (Yang y Nair, 2014). El turismo es servicio y hereda sus características de intangibilidad, heterogeneidad e inseparabilidad (Williams y Baláž, 2013) por lo que los consumidores de servicios perciben mayores riesgos que los consumidores de bienes (Mitchell y Greatorex, 1993b).

El turista se enfrenta a riesgos desde que comienza a organizar su viaje, cuando un turista compra un vuelo o reserva el alojamiento necesariamente está corriendo un riesgo puesto que está comprando un servicio intangible y una experiencia que sólo será experimentada después de haber sido consumida. Y una vez llega al destino se enfrenta tanto a riesgos directos como indirectos. A continuación, se expondrá una breve revisión sobre el riesgo percibido en turismo y sus tipos.

\subsection{Riesgo y riesgo percibido}

El riesgo es la probabilidad de que un incidente tenga consecuencia negativa en el comportamiento del consumidor (Rittichainuwat y Chakraborty, 2009, Laws y Prideaux, 2005; Glaesser, 2003). En la vida cotidiana existen muchos tipos de riesgos, riesgos generales que afectan tanto a los turistas como a los residentes en un destino, como la delincuencia, la salud y los desastres naturales. Pero los turistas están expuestos a riesgos específicos como las barreras culturales y de idioma, la hostilidad de los residentes, la comida local, el mal tiempo, las huelgas, el terrorismo, la falta de higiene, el fraude o la incertidumbre derivada de las leyes y reglamentos en el destino (Chiu y Lin, 2011; 
Reichel, Fuch y Uriely, 2007; Glensor y Peak, 2004; Zheng y Zhang, 2002; Sönmez y Graefe, 1998; Hauber y Zandbergen, 1996).

Pero cuando el turista viaja no realiza un mero viaje físico, el verdadero viaje es interior, radica en cómo percibe el destino. Por lo general, los turistas toman sus decisiones de viaje basadas en percepciones y no en la realidad (Reisinger y Mavondo, 2005; Roehl y Fesenmaier, 1992). El riesgo percibido es la percepción del consumidor de que ese incidente que tendrá consecuencias negativas en su comportamiento, vaya más allá de un nivel aceptable de tolerancia (Mansfeld y Pizam, 2006, Reichel, Fuchs, y Uriely, 2007). Tan pronto como el consumidor experimenta un cierto nivel de riesgo, su comportamiento cambia, retrasando la compra o utilizando estrategias diseñadas para reducir el nivel de riesgo hasta un nivel "tolerante" (Mowen y Minor 1998; Roselius, 1971). Por ejemplo, a través de la búsqueda de información o comprando una marca conocida (Reiche et al., 2007).

Al ser el riesgo percibido, los problemas de seguridad a menudo dependen del destino (Simpson y Siguaw, 2008; Kozak, Crotts y Law, 2007; Fuchs y Reichel, 2006; Carter, 1998) y de la imagen de seguridad del lugar, ya que el riesgo puede ser tanto percibido como no percibido por el turista. El riesgo percibido normalmente influye en las decisiones del consumidor, incluso si no existen en la realidad. Sin embargo, el riesgo no percibido no afecta el comportamiento del consumidor, aunque sea real y tangible (Simpson y Siguaw, 2008; Kozak et al., 2007; Fuchs y Reichel, 2006).

Entender el proceso de formación del riesgo percibido es importante para gestionar los destinos turísticos de manera sostenible y competitiva, ya que el riesgo percibido influye tanto en la imagen del destino (Rittichainuwat y Chakrabarti, 2009; Qi et al., 2009; Leppy Gibson, 2003; Lepp et al., 2011; Sönmez, y Graefe, 1998) e incluso en la imagen a través de la experiencia del turista en el destino (Carballo et al., 2019; Carballo y León, 2018; Carballo, Carballo y León, 2018; Fuentes, 2015), como en el comportamiento del turista (Kozak et al., 2007; Mansfeld, 2006; Sasso, 2005; Maser y Weiermair, 1998). Existen evidencias de la disminución en el número de llegadas de turistas sobre todo después de incidentes terroristas o de amenazas contra la seguridad, la recesión del turismo después de un ataque terrorista es bastante natural y ha sido examinada empíricamente como en el caso de la primavera árabe (Araña y León, 2008).

La necesidad de seguridad y viajes sin estrés será la clave que determine el comportamiento de muchos turistas. La cuestión de la seguridad plantea preguntas tan importantes como si los turistas internacionales perciben el riesgo de viaje, y qué factores influyen en sus percepciones del riesgo de viaje en los diferentes tipos de destino.

\subsection{Tipos de riesgo percibido asociados al turismo}

Muchos investigadores han puesto de manifiesto que el riesgo percibido por los turistas se encuentra en función de varios tipos de riesgos. Así, por ejemplo, Roehl y Fesenmaier (1992) identificó tres tipos riesgos percibidos a los que se enfrenta el turista al elegir un destino: riesgo neutral, riesgo funcional y riesgo del lugar. Mientras que Sönmez y Graefe (1998) identificaron diez tipos de riesgo asociado con los viajes internacionales: funcional, financiero, salud, físico, inestabilidad política, psicológico, satisfacción, social, terrorismo, y tiempo. Leppy Gibson (2003) identificaron siete factores de riesgo relacionados con las 
percepciones de riesgo en los jóvenes asociados con los viajes internacionales: la salud, la inestabilidad política, el terrorismo, los alimentos extraños, las barreras culturales, el dogma político y religioso de una nación y el crimen. Reisinger y Mavondo (2005) ampliaron este trabajo añadiendo factores de riesgo adicionales, utilizaron 13 tipos de riesgo turístico que van desde el cultural, funcional, financiera, salud, físico, político, psicológico, satisfacción, social, secuestros de aviones, explosión de una bomba, ataque bioquímico y de tiempo. Reichel et al., (2007) identificaron ocho tipos de riesgos percibidos relacionados con el tipo de viaje: riesgo físico relacionado con el lugar, socio-psicológico, daño físico, riesgo de expectativas, dificultades sociopolíticas, financiero, muchedumbre y de comportamiento. Qi et al (2009) exponen siete tipos de riesgo relacionados con la literatura de comportamiento del consumidor: riesgo de equipo, riesgo financiero, riesgo físico, riesgo psicológico, riesgo de satisfacción, riesgo social, y riesgo de tiempo. Karamustafa, Fuchs y Reichel (2013) también investigaron las percepciones de riesgo, incluyendo: riesgo social, financiero, psíquico, situaciones de presión, relacionado con la experiencia y de tiempo, y hotel. Más recientemente, Carballo, León y Carballo (2017) clasificaron los riesgos en salud, delincuencia, accidente, medioambiental y catástrofe, como los riesgos más importantes en el riesgo percibido por los turistas internacionales.

Todos estos riesgos percibidos se encuentran conectados, generando una imagen general de riesgo percibido del destino, y guiando las decisiones de viaje de los turistas. En este trabajo se proponen analizar los riesgos más comunes que pueden influir en el proceso de formación del riesgo percibido en de los destinos seleccionados (figura 1).

\section{Figura 1 \\ MODELO TEÓRICO PROPUESTO}

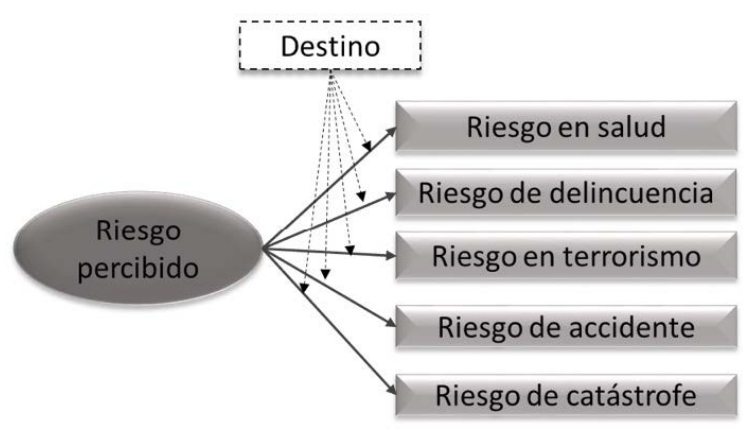

\section{METODOLOGÍA}

El estudio empírico para analizar los objetivos propuestos se realizó a través de un cuestionario diseñado para analizar el proceso de formación del riesgo percibido (Carballo, León y Carballo, 2017; Karamustafa et al., 2013). El trabajo de campo se realizó vía internet a través de una empresa especializada durante los meses de julio a septiembre de 2017. El cuestionario se suministró en Reino Unido y Alemania, por encontrarse ambos 
entre los principales países emisores de turistas en el mundo y especialmente en Canarias (Eurostat, 2019). Se han obtenido un total de 609 encuestas útiles (303 encuestas en Reino Unido y 306 en Alemania). La selección muestral se llevó a cabo con el fin de cumplir con una serie de cuotas en relación con las variables sociodemográficas: sexo, edad y educación, y de esta forma se garantiza que la muestra fuera representativa de la población. Por último, se seleccionaron a personas que habían realizado viajes al extranjero en los últimos dos años, hubiesen realizado o no visita previa a los destinos seleccionados. Los resultados descriptivos de clasificación de la muestra se reflejan en la siguiente tabla.

\section{Cuadro 1}

\section{PERFIL DE LOS ENCUESTADOS}

\begin{tabular}{lll}
\hline Variables & ReinoUnido & Alemania \\
\hline Total encuestas & 303 & 306 \\
Genero\% & & \\
Hombre & 50 & 50 \\
Mujer & 50 & 50 \\
Edad\% & & \\
16-24 & 20 & 20 \\
25-34 & 20 & 20 \\
35-44 & 20 & 20 \\
45-54 & 20 & 20 \\
55-64 & 11 & 11 \\
Más de 64 & 9 & 9 \\
& & \\
Nivel educativo $\%$ & & 12 \\
Primaria & 1 & 47 \\
Secundaria & 29 & 20 \\
Grado Medio & 16 & 21 \\
Grado Superior & 54 & \\
\hline
\end{tabular}

En el cuestionario se propusieron tres destinos turísticos: Canarias (España), Brasil y Colombia. En la primera parte del cuestionario se preguntaba sobre la probabilidad percibida de sufrir cinco tipos de riesgos en el destino en una escala Likert de 7 puntos ( $7=$ muy probable, $1=$ poco probable): El riesgo de salud (contraer una enfermedad por ingerir algún alimento o bebida, enfermedad de transmisión animal (malaria, etc...) ), el riesgo de delincuencia (sufrir un asalto con violencia física), riesgo de terrorismo (que ocurra un ataque terrorista), riesgo de accidente (sufrir un accidente de tráfico) y riesgo de catástrofe (que ocurra un desastre natural (Terremotos, tormentas, tsunamis, erupciones volcánicas, incendios forestales...).

El tratamiento estadístico de los datos se realizó con el paquete estadístico AMOS 24 y se empleó un análisis SEM para establecer la relación entre la percepción del riesgo y 
los diferentes tipos de riesgo que se pueden encontrar en el destino. Además de probar el modelo teórico, el estudio también probó los efectos moderadores del destino sobre las relaciones causales entre la percepción del riesgo y los tipos de riesgos.

\section{RESULTADOS}

\subsection{Análisis de las relaciones causales propuestas}

El modelo SEM es un método apropiado para evaluar el ajuste de modelos que contiene indicadores (o variables observadas) y variables latentes (no observables) a los datos recopilados, determinando la validez y fiabilidad de las medidas (Jorskog y Sorbom, 1989). El modelo propuesto tiene como finalidad estimar las relaciones entre la percepción del riesgo como variables latentes y los diferentes tipos de riesgos como atributos(variables observables).

El análisis de regresión múltiple se realizó mediante el proceso de Máxima Verosimilitud (Prayag, 2009). El estadístico Chi-cuadrado fue significativo $\left(\mathrm{X}^{2}=33\right.$. 3, gl=12, $\mathrm{p}<0$ ) para un nivel de significación del 95\%, y esto se explica porque este estadístico es sensible al tamaño de la muestra. En su lugar, la relación $\mathrm{X}^{2} / \mathrm{gl}\left(\mathrm{X}^{2} / \mathrm{gl}=2\right.$. 1) es mejor indicador, un valor menor que 3 se considera aceptable (Prayag, 2009). Pero un solo estadístico no es suficiente para establecer el ajuste del modelo, en su lugar hay que tener en cuenta otros índices como los índices de ajuste y el error cuadrático medio de la aproximación (RMSEA). Los índices de ajuste dieron todos mayores de 0.9 (CFI=.996; TLI=.991; IFI=.996; RFI=.983; NFI=.993, GFI=994, AGFI=.978) lo que demuestra que el modelo plateado se ajusta bien a los datos empíricos. El error cuadrático medio de la aproximación (RMSEA) fue 0.072, menor que el valor máximo de 0.08 que se considera para un buen nivel de ajuste para el modelo (Chen y Chou, 2019). Por lo tanto, el modelo presenta un ajuste aceptable. Los resultados de la estimación se muestran en el modelo estructural con los coeficientes estandarizados (tabla 2). Todos los resultados fueron significativos para un nivel de significación del 5\% (0.05). Esto sugiere que el objetivo planteado se alcanza con el modelo formulado. Concretamente, el modelo muestra que los distintos tipos de riesgos contribuyen de forma positive y significativa a la formación del riesgo percibido (se ha dejado el riesgo de catástrofe como variable de referencia), por lo que aceptamos las cuatro hipótesis propuestas. Además, cada uno de los estimadores explican la importancia relativa de las variables explicativas(tipos de riesgos) en contribución a la variable latente (riesgo percibido). Así, podemos afirmar que el riesgo percibido en el destino depende significativamente del riesgo en salud $(\beta=.82 ; p<.000)$ con una importancia relativa superior. Seguidamente, son significativos los riesgos de delincuencia $(\beta=.73 ; \mathrm{p}<.000)$ y terrorismo $(\beta=.70, \mathrm{p}=0.0)$ con una importancia muy similar en el riesgo final percibido por los turistas. Sin embargo, el riesgo de accidente $(\beta=.47, \mathrm{p}=0.0)$ tiene una importancia relativa muy inferior en la formación del riesgo percibido. 


\section{Cuadro 2 \\ ESTIMADORES ESTANDARIZADOS DEL MODELO ESTRUCTURAL}

\begin{tabular}{llll}
\hline Relaciones causales & & & \\
ML $(95 \%)$ & Estimadores & P & Resultados \\
$(606)$ & .82 & .000 & Se acepta \\
\hline Riesgo percibido $\rightarrow$ Riesgo en salud & .73 & .000 & Se acepta \\
Riesgo percibido $\rightarrow$ Riesgo de delincuencia & .70 & .000 & Se acepta \\
Riesgo percibido $\rightarrow$ Riesgo en terrorismo & .47 & .000 & Se acepta \\
Riesgo percibido $\rightarrow$ Riesgo de accidente & & & \\
\hline
\end{tabular}

\subsection{Análisis multigrupo}

Además de probar el modelo teórico, este trabajo también analizó los efectos moderadores de la variable "destino" en cada una de las relaciones propuesta en el modelo. Para ello, se dividió la muestra en dos subgrupos según los destinos; Europa (Canarias) y Latinoamérica (Colombia y Brasil). El tamaño de los grupos fue de 203 y 406, respectivamente.

El objetivo del análisis multigrupo es examinar cómo las relaciones entre el riesgo percibido y los tipos de riesgos varían entre los grupos de destinos (Bou-Llusar et al., 2016; Cupani y Pautassi, 2013; Bou y Satorra, 2010; Byrne, 2001). Es decir, los distintos tipos de riesgos afectan de distinta manera dependiendo del destino visitado, de manera que podemos encontrar determinados riesgos que se perciban más peligrosos en un destino que en otro.

Para ello, el análisis multigrupo se realiza en dos pasos. En primer lugar, se realiza la comparación de los grupos en el mismo modelo especificando la restricción de igualdad entre grupos (Modelo Restringido). De este modo, se genera un valor global de Chi-cuadrado para los conjuntos de submodelos como parte de un único sistema estructural. Posteriormente, se especifica el modelo sin restricción de igualdad entre grupos, estimándose los parámetros libremente (Modelo no restringido), lo que da como resultado un segundo valor de Chi-cuadrado normalmente con menos grados de libertad. Las diferencias significativas en los valores de Chi-cuadrado entre los dos modelos determinan el efecto moderador. Si el cambio en el valor de Chi-cuadrado es estadísticamente significativo, la hipótesis nula de la invarianza de parámetro se rechaza y se acepta por tanto el efecto moderador (Suki, 2014; Byrne, 2004; Carballo, León y Carballo, 2020). La interpretación de estos resultados produce una gran cantidad de información interesante.

Primero, se analiza el efecto moderador del destino en todas las relaciones causales simultáneamente, comparando el modelo con restricción y sin restricción. Los resultados (tabla 3) muestran una diferencia significativa en la variación de la Chi-cuadrado entre los dos modelos $\left(\Delta X^{2}=15.1, \mathrm{p}<0\right)$ para un nivel de significación del $5 \%$. Por lo que rechazamos la hipótesis nula de la invarianza, lo que significa que al menos en una de las relaciones causales propuestas en el modelo varía en función del destino. Posteriormente, se analiza 
el efecto moderador del destino por separado para cada una de las relaciones causales comparando el modelo con y sin restricciones.

La Tabla 3 muestra los resultados del análisis multigrupo. Cada uno de los coeficientes beta explicaba la importancia relativa de los tipos de riesgos en su contribución a la percepción de riesgo. Parece que los grupos de destinos moderaron significativamente las relaciones causales entre el riesgo en salud y la percepción de riesgo $\left(\Delta X^{2}=14.8, p<0\right)$. Curiosamente, la relación entre el riesgo en la salud y la percepción de riesgo es significativa para ambos destinos $(\mathrm{p}<0)$ y se muestra más fuerte en los países Latinoamericanos $(\beta=.86)$ que en Europa $(\beta=.64)$. Los resultados también muestran que los grupos de destino moderan significativamente la relación entre riesgo en delincuencia y la percepción del riesgo $\left(\Delta X^{2}=7.9, p<0\right)$. El riesgo de delincuencia es también significativo para cada uno de los destinos analizados y tiene mayores efectos sobre la percepción de riesgo en Latinoamérica $(\beta=.73)$ que en Europa $(\beta=.55)$. Con respecto a la relación del riesgo en terrorismo y la percepción del riesgo, la tipología de destino también ejerce un efecto significativo y mayor en $\operatorname{Europa}(\beta=.76)$ que en Latinoamérica $(\beta=.59)$. Sin embargo, los resultados muestran que los grupos de destino no moderaron significativamente la relación entre el riesgo de accidente y la percepción del riesgo $\left(\Delta X^{2}=.53, p>0\right)$ por lo que el riesgo de accidente no se percibe diferentes para cada destino.

\section{Cuadro 3 \\ ESTIMADORES ESTANDARIZADOS DEL MODELO MULTIGRUPO. DESTINOS}

\section{Comparación de modelos}

\begin{tabular}{llll}
\hline $\begin{array}{l}\text { Relaciones causales } \\
\text { ML }(95 \%)\end{array}$ & $\Delta X^{2}$ & Latinoamérica & Europa \\
$(609)$ & $15.1^{*}$ & & \\
\hline General & $14.8^{*}$ & $.86^{*}$ & $.64^{*}$ \\
$\begin{array}{l}\text { Percepción de Riesgo } \rightarrow \text { Riesgo en salud } \\
\text { Percepción de Riesgo } \rightarrow \text { Riesgo de delincuencia }\end{array}$ & $7.9^{*}$ & $.73^{*}$ & $.55^{*}$ \\
$\begin{array}{l}\text { Percepción de Riesgo } \rightarrow \text { Riesgo en terrorismo } \\
\text { Percepción de Riesgo } \rightarrow \text { Riesgo de accidente }\end{array}$ & $6.04^{*}$ & $.59^{*}$ & $.76^{*}$ \\
\hline
\end{tabular}

*Significant $(\mathrm{p}<.05)$

\section{CONCLUSIONES}

Algunas investigaciones muestran como la información sobre riesgo en los destinos turísticos, ya sea a través de los medios de comunicación (Alvarez y Campo, 2014; Rittichainuwat y Chakraborty, 2009; Tasci y Gartner, 2007; Sönmez y Graefe, 1998) o de boca en boca (Rittichainuwat y Chakraborty, 2009; Cavlek, 2002) aumentan la percepción de los turistas sobre el riesgo. Sin embargo, existe aún un importante desconocimiento sobre 
cuáles son las tipologías de riesgo que conforman esa percepción general de seguridad en los destinos turísticos, y el peso de cada variable en dicha percepción de seguridad.

La seguridad es uno de los aspectos más importantes que define la calidad de los servicios prestados en los destinos turísticos (Donaldson y Ferreira, 2009). La comprensión de las percepciones de los turistas sobre la seguridad en un destino es importante para crear un entorno favorable para el desarrollo del turismo.

Este trabajo ha analizado la influencia que los distintos tipos de riesgo ejercen en la percepción de seguridad. En concreto, el riesgo de salud es el que ejerce una mayor influencia en el riesgo percibido. Esto denota la importancia de los riesgos sanitarios (malestares gástricos, posibles enfermedades infecciosas, etc. ) donde la elevada probabilidad de ocurrencia pondera de forma significativamente el impacto sobre el riesgo percibido. Los riesgos de delincuencia y terrorismo muestran pesos similares en la percepción de seguridad de los destinos. Si bien el terrorismo tiene unas motivaciones bien diferenciadas de la delincuencia, su influencia en el riego percibido es similar.

Por último, el riesgo de accidente es el que presenta un menor impacto en la conformación del riesgo percibido. Con independencia de los datos objetivos de probabilidad de ocurrencia e impacto del riesgo, es evidente que la percepción del riesgo es un aspecto subjetivo del turista.

En relación al efecto moderador del tipo de destino en el riesgo percibido, o cómo cada destino en particular afecta la percepción de seguridad en la mente del turista, los resultados muestran que los distintos factores tanto en salud como en delincuencia, parecen presentar una mayor incidencia en los destinos Latinoamericanos que en Europa. El riesgo de accidente no se encuentra moderado por el destino. Curiosamente, el riesgo de terrorismo es mayor en Europa que en los países latinoamericanos, esto puede deberse a que, en la última década, la frecuencia de los ataques letales tiende a ser mayor en las zonas económicamente más desarrolladas, cerca de las grandes ciudades y dentro de los países democráticos (Python et al., 2016). Europa Occidental han sido el escenario de algunos de los actos terroristas más crueles, y en dos ocasiones se superaron las 100 víctimas mortales en actos terroristas: en los atentados de Atocha en Madrid (marzo 2004; 191 víctimas), y en Paris (noviembre 2015; 132 víctimas). El atentado en Londres (julio 2005; 56 víctimas) también fue especialmente destacable en número de víctimas. Europa uno de los lugares más seguros del planeta, más seguro que hace décadas, pero lo que determina las decisiones de viaje son las percepciones de riesgo.

Como implicaciones de este trabajo cabe señalar que la percepción del riesgo en los destinos turísticos es subjetiva y tanto a la imagen como a las intenciones de visitar el destino (Carballo, Carballo y León, 2017; Carballo et al., 2015; Carballo, 2015; Hultman, Skarmeas, Oghazi y Beheshti, 2015; Qu, Hyunjung y Hyunjung, 2011). Sería aconsejable que los destinos turísticos proyectasen una imagen incorporando los aspectos discutidos previamente a la hora de realizar estrategias de marketing, en función del peso de cada tipo de riesgo. El riesgo en salud tiene mucha importancia en la formación del riesgo percibido, por lo que es importante proyectar los aspectos positivos de los servicios médicos y de emergencias en un destino, sobre todo en Latinoamérica, donde se percibe este riesgo como más peligroso que en Europa. En el caso de Europa, aunque en menor proporción también tiene gran importancia. Por otro lado, mientras que en Latinoamérica también 
debe prestarse mucha atención al riesgo de delincuencia, en Europa las estrategias deben basarse en los aspectos de seguridad en riesgo sobre terrorismo ya que este no solo afecta a la imagen del destino europeo sino también a su demanda (Araña y León, 2008).

Por último, y como limitaciones del estudio, cabe plantear si los destinos latinoamericanos seleccionados (Brasil y Colombia) y el destino europeo (Canarias) con una condición insular y alejada del continente, influyen en los resultados, siendo recomendable hacer análisis posteriores en otros destinos alternativos. Por otra parte, también los mercados de origen analizados (Alemania y Reino Unido) condicionan estos resultados y sus implicaciones. Sería también aconsejable que las encuestas se realizaran teniendo en cuenta si el individuo ha realizado visitas previas al destino y por lo tanto ya tiene experiencia en el destino, esto podría influir en los resultados. Además, las futuras líneas de investigación también pueden analizar como las características de los individuos (demográficas, motivaciones de viaje, tipográficas, etc. ) influyen en el riesgo percibido.

\section{BIBLIOGRAFÍA}

ALVAREZ, M. D. y CAMPO, S. (2014): «The influence of political conflicts on country image and intention to visit: A study of Israel's image», Tourism Management, vol. 40, pp. 70-78.

ARAÑA, J. y LEÓN, C. (2008): "The Impact of Terrorism on Tourism Demand", Annals of Tourism Research, vol. 25 (2), pp. 299-315.

BOU, J. C. y SATORRA, A. (2010): «A multigroup structural equation approach: A demonstration by testing variation of firm profitability across EU samples», Organizational Research Methods, vol. 13 (4), pp. 738-766.

BOU-LLUSAR, J. C., BELTRÁN-MARTÍN, I., ROCA-PUIG, V. y ESCRIG-TENA, A. B. (2016): «Single-and multiple-informant research designs to examine the human resource management- performance relationship», British Journal of Management, vol. 27(3), pp. 646-668.

BYRNE, B. M. (2001): «Structural equation modeling with AMOS, EQS, and LISREL: Comparative approaches to testing for the factorial validity of a measuring instrument», International Journal of Testing, vol. 1(1), pp. 55-86.

BYRNE, B. M. (2004): «Testing for multigroup invariance using AMOS graphics: A road less traveled», Structural Equation Modeling, vol. 11 (2), pp. 272-300.

CARBALLO FUENTES, R. (2015): «Tourist experience, image, security and cultural centres», European Journal of Tourism Research, vol. 11, pp. 189-193.

CARBALLO M. M., ARAÑA, J. E., LÉON C. J. y MORENO-GIL, S. (2015): «Economic valuation of tourism destination image», Tourism Economics, vol. 21 (4), pp. 741-759

CARBALLO, R. R., LEÓN, C. J. y CARBALLO, M. M. (2017): «The perception of risk by international travelers». Worldwide Hospitality and Tourism Themes, vol. 9 (5), pp. 534-542.

CARBALLO, R. R. y LEÓN, C. J. (2018): «The influence of artistically recreated nature on the image of tourist destinations: Lanzarote's art, cultural and tourism visitor centres and their links to sustainable tourism marketing». Journal of Sustainable Tourism, vol. 26 (2), pp. 192-204. 
CARBALlO, R. R., CARBALlO, M. M. y LEÓN, C. J. (2018): «3 The tourist centres' image», in Green Events and Green Tourism: An International Guide to Good Practice.

CARBALLO, R., LEÓN, C. y CARBALLO, M: (2019): «Fighting Overtourism at Lanzarote (Spain)».Worldwide Hospitality and Tourism Themes, vol. 11 (5), pp. 506-515.

CARBALLO, R., LEÓN, C. y CARBALLO, M. (2020): «6 Training of human resources in tourism to mitigate overtourism and promote a sustainable destination», in Overtourism and Tourism Education: A Strategy for Sustainable Tourism Futures, 96.

CARTER, S. (1998): «Tourists and Traveler's Social Construction of Africa and Asia as Risky Locations», Tourism Management, vol. 19, pp. 349-358.

CAVLEK, N. (2002): «Tour operators and destination safety», Annals of Tourism Research, vol. 29 (2): pp. 478-496.

CHEN, C. F. y CHOU, S. H. (2019): «Antecedents and consequences of perceived coolness for Generation $\mathrm{Y}$ in the context of creative tourism-A case study of the Pier 2 Art Center in Taiwan», Tourism Management, vol. 72, pp. 121-129.

CHIU, S. P. y LIN, S. Y. (2011): «Study on risk perceptions of international tourists in India», African Journal of Business Management, vol. 5 (7), pp. 2. 742-2. 752.

CUPANI, M. y PAUTASSI, R. M. (2013): «Predictive contribution of personality traits in a sociocognitive model of academic performance in mathematics», Journal of Career Assessment, vol. 21 (3), pp. 395-413.

DONALDSON, R. y FERREIRA, S. (2009): «(Re-) creating urban destination image: Opinions of foreign visitors to South Africa on safety and security?», Urban Forum, vol. 20 (1), p. 1.

EUROSTAT (2019): Disponible en https://appsso.eurostat.ec.europa.eu/nui/show. do?dataset=tour_dem_ttw\&lang=en

FUENTES, R. C., MORENO-GIL, S., GONZÁLEZ, C. L. y RITCHIE, J. B. (2015): "La creación y promoción de experiencias en un destino turístico. Un análisis de la investigación y necesidades de actuación, Cuadernos de Turismo, n 35, pp. 71-94.

FUCHS, G. y REICHEL, A. (2006): «Tourist destination risk perception: The case of Israel», Journal of Hospitality \& Leisure Marketing, vol. 14 (2), pp. 83-108.

GLAESSER, D. (2003): Crises' spheres of activity. Crisis management in the tourism industry, Burlington, MA: Elsevier Butterworth-Heinemann.

GLENSOR, R. W. y PEAK, K. J. (2004): Crimes against tourists, US Department of Justice, Office of Community Oriented Policing Services.

HAUBER, A. R. y ZANDBERGEN, A. G. (1996): «Foreign visitors as targets of crime in the Netherlands: perceptions and actual victimization over the years 1989, 1990, and 1993», Security Journal, vol. 7 (3), pp. 211-218.

HULTMAN, M., SKARMEAS, D., OGHAZI, P. y BEHESHTI, H. M. (2015): «Achieving tourist loyalty through destination personality, satisfaction, and identification», Journal of Business Research, vol. 68 (11), pp. 2. 227-2. 231.

JORSKOG, K. G. y D. SORBOM (1989): LISREL 7-A guide to the program and applications, edited by Chicago: SPSS Publications.

KARAMUSTAFA, K., FUCHS, G. y REICHEL, A. (2013): «Risk perceptions of a mixedimage destination: The case of Turkey's first-time versus repeat leisure visitors», Journal of Hospitality Marketing \& Management, vol. 22 (3), pp. 243-268. 
KOZAK, M., CROTTS, J. C. y LAW, R (2007): «The impact of the perception of risk on international travelers», The International Journal of Tourism Research, vol. 9 (4), pp. 233-242.

LAWS, E. y PRIDEAUX, B. (2005): «Crisis management: a suggested typology», Journal of Travel and Tourism Marketing, vol. 19 (2/3), pp. 1-8.

LEPP, A. y GIBSON, H. (2003): «Tourist roles, perceived risk and international tourism», Annals of Tourism Research, vol. 30 (3), pp. 606-624.

LEPP, A., GIBSON, H. y LANE, C. (2011): «Image and perceived risk: A study of Uganda and its official tourism website», Tourism Management, vol. 32 (3), pp. 675-684.

MANSFELD, Y. y PIZAM, A. (Eds. ). (2006): Tourism, security and safety, Routledge.

MASER, B. y WEIERMAIR, K. (1998): «Travel decision-making: From the vantage point of perceived risk and information preferences», Journal of Travel \& Tourism Marketing, vol. 7 (4), pp. 107-121.

MITCHELL, V. W. y GREATOREX, M. (1993): «Risk perception and reduction in the purchase of consumer services», Service Industries Journal, vol. 13 (4), pp. 179-200.

MOWEN, J. y MINOR, M. (1998): Consumer behavior. Englewood Cliffs, NJ: PrenticeHall.

OPPERMANN, M. (1998): «Destination threshold potential and the law of repeat visitation», Journal of Travel Research, vol. 37 (2), pp. 131-138.

OTTO, J. E. y RITCHIE, J. B. (1996): «The service experience in tourism», Tourism Management, vol. 17 (3), pp. 165-174.

PRAYAG, G. (2009): «Tourists' evaluations of destination image, satisfaction, and future behavioral intentions-The case of Mauritius», Journal of Travel \& Tourism Marketing, vol. 26 (8), pp. 836-853.

PYTHON, A., ILLIAN, J., JONES-TODD, C. y BLANGIARDO, M. (2016): A Bayesian approach to modelling fine-scale spatial dynamics of non-state terrorism: world study, 2002-2013. arXiv preprint arXiv: 1610. 01215.

QI, C., GIBSON, H. y ZHANG, J. (2009): «Perceptions of risk and travel intentions: the case of China and the Beijing Olympic Games», Journal of Sport \& Tourism, vol. 14 (1), pp. 43-67.

QU, H., HYUNJUNG, K. M. y HYUNJUNG, H. (2011): «A model of destination branding: Integrating the concepts of the branding and destination image», Tourism Management, vol. 32 (3), pp. 465-476.

REICHEL, A., FUCHS, G. y URIELY, N. (2007): «Perceived risk and the non-institutionalized tourist role: The case of Israeli student ex-backpackers», Journal of Travel Research, vol. 46 (2), pp. 217-226.

REICHEL, A., FUCHS, G. y URIELY, N. (2007): «Perceived risk and the non-institutionalized tourist role: The case of Israeli student ex-backpackers», Journal of Travel Research, vol. 46 (2), pp. 217-226.

REISINGER, Y. y MAVONDO, F. (2005): «Travel anxiety and intentions to travel internationally: Implications of travel risk perception», Journal of Travel Research, vol. 43 (3), pp, 212-225. 
RITTICHAINUWAT, B. N. y CHAKRABORTY, G. (2009): «Perceived travel risks regarding terrorism and disease: The case of Thailand», Tourism Management, vol. 30 (3), pp. 410-418.

ROEHL, W. S. y FESENMAIER, D. R. (1992): «Risk perceptions and pleasure travel: An exploratory analysis», Journal of Travel Research, vol. 30 (4): pp. 17-26.

ROSELIUS, T. (1971): «Consumer rankings of risk reduction methods», Journal of Marketing, vol. 35 (1), pp. 56-61.

SASSO, S. (2005): «Perceived risk», International Encyclopedia of Hospitality Management, pp. 465

SIMPSON, P. M. y SIGUAW, J. A. (2008): «Perceived travel risks: The traveler perspective and manageability», International Journal of Tourism Research, vol. 10 (4), pp. 315-327.

SÖNMEZ, S. F. y GRAEFE, A. R. (1998): «Influence of terrorism risk on foreign tourism decisions. Annals of Tourism Research, vol. 25 (1), pp. 112-144.

SUKI, N. M. (2014): «Passenger satisfaction with airline service quality in Malaysia: A structural equation modeling approach», Research in Transportation Business \& Management, vol. 10, pp. 26-32.

TASCI, A. D y GARTNER, W. C. (2007): «Destination image and its functional relationships», Journal of Travel Research, vol. 45 (4), pp. 413-425.

WILLIAMS, A. M. y BALÁŽ, V. (2013): «Tourism, risk tolerance and competences: Travel organization and tourism hazards», Tourism Management, vol. 35, pp. 209-221.

WILLIAMS, A. M. y BALÁŽ, V. (2015): «Tourism risk and uncertainty: Theoretical reflections», Journal of Travel Research, vol. 54 (3), pp. 271-287.

YANG, E. C. L y NAIR, V. (2014): «Tourism at risk: A review of risk and perceived risk in tourism», Asia-Pacific Journal of Innovation in Hospitality and Tourism (APJIHT), vol. 3 (2), pp. 1-21.

ZHENG, X. y ZHANG, J. F. (2002): Travel safety theory and practice: case study of Fujian Province, China, Hong Kong: Hong Kong Education and Social Scientific Research Society 